\title{
Evaluation of efficiency of hemi-nested PCR assay for the detection of 'Candidatus Liberibacter' infecting citrus
}

\author{
Adpangaya Sahana Bhaskara', Bandalli N Jawad Ahmed², Charith R Adkar-Purushothama3,\#, Mysore N Nagendra Prasad3, \\ Marikunte Y Sreenivasa' \& Pavagada K Murthy Maheshwar2.* \\ 1 Department of Studies in Microbiology, University of Mysore, Mysore 570 006, Karnataka, India \\ 2 Department of Microbiology, Yuvaraja's College, University of Mysore, Mysore 570 005, Karnataka, India \\ 3 Department of Biotechnology, Sri Jayachamarajendra College of Engineering, Mysore 570 006, Karnataka, India \\ \# Present address: Université de Sherbrooke, Faculté de médecine et des sciences de la santé, Département de biochimie, \\ Pavillon de recherche appliquée sur le cancer (PRAC), 3201, rue Jean-Mignault, Sherbrooke, Québec, Canada \\ * Corresponding author: pkmaheshwaragmail.com
}

Received 20 March 2013, accepted 02 September 2013

\begin{abstract}
The present study reports the development and evaluation of a hemi-nested polymerase chain reaction (hnPCR) assay for the efficient detection of 'Candidatus Liberibacter' infecting citrus plants. A set of new primers was designed by alignment of nucleotide sequences of the $\beta$-operon (rplKAJLrpoBC) ribosomal protein genes from all the known 'Candidatus Liberibacter asiaticus' isolates reported in Genbank. Hemi-nested PCR reaction components and thermal cycling parameters were optimized and reaction conditions were standardized. Sequencing of the PCR products from hemi nested-PCR reactions confirmed the specificity of new primer pairs for 'Candidatus Liberibacter asiaticus'. The reliability and sensitivity of hnPCR was evaluated by comparing it to real-time PCR.
\end{abstract}

Key words: hnPCR, Huanglongbing, Liberibacter, ribosomal protein gene

\section{Introduction}

Huanglongbing (HLB) disease is a serious threat to citrus production and continues to invade new citrus-growing areas (Bové 2006). The causative agent of this disease is a phloem-restricted, fastidious, Gram-negative bacterium of the genus 'Candidatus Liberibacter' (Ca. Liberibacter), placed in the $\alpha$-Proteobacteria subdivision (Jagoueix et al. 1994). This pathogen has been reported from Asia (' $\mathrm{Ca}$. L. asiaticus'), Africa (' $\mathrm{Ca}$. L. africanus') and the American continents (' $\mathrm{Ca}$. L. americanus' and 'Ca. L. asiaticus). Symptoms of this disease are development of yellow shoots, bearing a few small-sized, deformed and poorly coloured fruits with greening appearance (Bové 2006). Further blotchy mottle of leaves, that leads to the development of yellow shoots; tree stunting and declining are also observed. It is also reported that ' $\mathrm{Ca}$. Liberibacter' affects roots of citrus plants which in turn start decaying from the rootlets (Graça da 1991).

Transmission of ' $\mathrm{Ca}$. Liberibacter' is mainly through infected budwood and vectors (psyllid), which can be man- aged by the propagation of clean nursery stock and vector control. However, trees in commercial citrus groves and private yards need to be tested regularly by a sensitive and reliable technique, so that infected trees can be removed to prevent further spread of the disease (Urasaki et al. 2008). Various methods such as electron microscopy (Catling et al. 1978), dot-blot hybridization (Varma et al. 1993), polymerase chain reaction (Jagoueix et al. 1996, Hocquellet et al. 1999, Fujikawa \& Iwanami 2012), direct PCR from the mid-rib extract (Fujikawa et al. 2013), nested PCR (Ding et al. 2005), long PCR (Hoy et al. 2001), duplex conventional PCR (Donnua et al. 2012), reverse transcription-duplexPCR (Adkar-Purushothama et al. 2010), real-time PCR (Li et al. 2006, Pietersen et al. 2010, Morgan et al. 2012), and cycleave isothermal and chimeric primer-initiated amplification of nucleic acids (Cycleave ICAN; Urasaki et al. 2008) have been developed for the diagnosis of this phytopathogenic bacterium. Currently, because of the increased sensitivity, reproducibility and the reduced risk of carry-over contamination, real-time PCR (qPCR) has gained popularity over conventional PCR and other molecular based detection techniques (Mackay et al. 2002, Loconsole et al. 2010).

In these circumstances, a specific and cost-effective alternative technique will be helpful for routine diagnosis and management of HLB. In this regard, hemi-nested PCR (hnPCR) described in the present study could be a useful alternative technique for the detection of ' $\mathrm{Ca}$. Liberibacter asiaticus', for screening purposes and also for selecting healthy plant material for propagation.

\section{Materials and methods}

Plant sample and total nucleic acid extraction

Leaf samples were collected from trees of sweet orange (Citrus sinensis), mandarin (C. reticulate), key lime (C. aurantifolia), lemon $(C . \times$ limon) pomelo $(C$. maxima $)$ and citron (C. decumana) exhibiting HLB symptoms (30) and asymptomatic (85) from citrus orchards in India (Table 1). Total nucleic acid was extracted as described previously (Adkar-Purushothama et al. 2010). 
Table 1: Comparative results between conventional PCR, hnPCR and real-time PCR for the presence of 'Ca. L. asiaticus' in both disease symptomatic and asymptomatic citrus varieties: sweet orange (C. sinensis), mandarin (C. reticulate), key lime (C. aurantifolia), lemon (C. x limon), pomelo (C. maxima) and citron (C. decumana).

\begin{tabular}{|c|c|c|c|c|c|c|c|c|c|}
\hline $\begin{array}{l}\text { Place of collec- } \\
\text { tion/Sample ID }\end{array}$ & $\begin{array}{l}\text { Disease } \\
\text { symptom }\end{array}$ & $\begin{array}{l}\text { Conven- } \\
\text { tional PCR }\end{array}$ & hnPCR & $\begin{array}{l}\text { Real-time } \\
\text { PCR } \\
\text { Ct value* }\end{array}$ & $\begin{array}{l}\text { Place of collec- } \\
\text { tion/SampleID }\end{array}$ & $\begin{array}{l}\text { Disease } \\
\text { symptom }\end{array}$ & $\begin{array}{l}\text { Conven- } \\
\text { tional PCR }\end{array}$ & $\mathrm{hnPCR}$ & $\begin{array}{l}\text { Real-time } \\
\text { PCR } \\
\text { Ct value* }\end{array}$ \\
\hline \multicolumn{5}{|c|}{ Sweet orange trees (C. sinensis) } & \multicolumn{5}{|c|}{ Lime trees (C. aurantifolia) } \\
\hline Ammati-1 & - & - & + & 35.22 & Ammati-1 & - & - & - & 0 \\
\hline Ammati-2 & - & - & - & 0 & Ammati-2 & - & - & - & 0 \\
\hline Ammati-3 & + & + & + & 25.01 & Chikmagalur-1 & - & + & + & 29.53 \\
\hline Chikmagalur-1 & + & + & + & 24.05 & Chikmagalur-2 & + & + & + & 26.94 \\
\hline Chikmagalur-2 & + & + & + & 24.95 & Hassan-1 & - & - & - & 37.64 \\
\hline Hassan-1 & - & - & - & 39.05 & Hassan-2 & - & - & + & 35.68 \\
\hline Hassan-2 & + & + & + & 25.48 & Hassan-3 & - & - & + & 33.56 \\
\hline Gonikoppal-1 & - & + & + & 30.22 & Hassan-4 & + & + & + & 26.79 \\
\hline Gonikoppal-2 & + & + & + & 24.76 & Gonikoppal-1 & - & - & + & 34.37 \\
\hline Polibetta-1 & - & - & + & 34.01 & Gonikoppal-2 & - & - & - & 38.03 \\
\hline Polibetta-2 & - & + & + & 30.23 & Polibetta-1 & - & + & + & 35.02 \\
\hline Polibetta-3 & + & + & + & 26.92 & Polibetta-2 & - & - & - & 0 \\
\hline Polibetta-4 & + & + & + & 26.43 & Sakleshpur-1 & - & - & - & 37.58 \\
\hline Sakleshpur-1 & - & - & - & 0 & Sakleshpur-2 & - & + & + & 31.14 \\
\hline Sakleshpur-2 & + & + & + & 24.68 & Sakleshpur-3 & + & + & + & 25.01 \\
\hline Sakleshpur-3 & + & + & + & 26.33 & Siddapura-1 & - & - & - & 39.29 \\
\hline Siddapura-1 & - & - & - & 39.54 & Siddapura-2 & - & - & - & 0 \\
\hline Siddapura-2 & + & + & + & 25.92 & Somavarapete-1 & - & + & + & 30.41 \\
\hline Siddapura-3 & + & + & + & 25.64 & Somavarapete-2 & - & - & - & 37.73 \\
\hline Somavarapete-1 & - & - & - & 38.54 & & & & & \\
\hline Somavarapete-2 & - & - & - & 0 & & & & & \\
\hline Somavarapete-3 & + & + & + & 24.65 & & & & & \\
\hline \multicolumn{5}{|c|}{ Mandarin trees (C. reticulate) } & \multicolumn{5}{|c|}{ Lemon trees $(C . \times$ limon $)$} \\
\hline Ammati-1 & - & - & + & 33.86 & Ammati-1 & - & - & + & 35.06 \\
\hline Ammati-2 & - & - & - & 0 & Ammati-2 & + & + & + & 26.88 \\
\hline Ammati-3 & + & + & + & 25.48 & Chikmagalur-1 & - & - & - & 0 \\
\hline Chikmagalur-1 & - & - & - & 39.03 & Chikmagalur-2 & - & - & - & 39.88 \\
\hline Chikmagalur-2 & - & + & + & 32.16 & Chikmagalur-3 & + & + & + & 24.36 \\
\hline Hassan-1 & - & - & - & 0 & Hassan-1 & - & + & + & 29.45 \\
\hline Hassan-2 & - & + & + & 29.48 & Hassan-2 & - & - & - & 0 \\
\hline Hassan-3 & + & + & + & 25.94 & Hassan-3 & + & + & + & 24.58 \\
\hline Gonikoppal-1 & - & - & + & 35.62 & Gonikoppal-1 & - & - & + & 33.67 \\
\hline Gonikoppal-2 & - & - & - & 37.56 & Gonikoppal-2 & - & + & + & 31.29 \\
\hline Gonikoppal-3 & + & + & + & 24.67 & Polibetta-1 & - & + & + & 30.34 \\
\hline Polibetta-1 & - & + & + & 28.39 & Polibetta-2 & - & - & - & 37.45 \\
\hline Polibetta-2 & - & - & + & 34.83 & Sakleshpur-1 & - & - & - & 38.54 \\
\hline Polibetta-3 & - & - & + & 35.92 & Sakleshpur-2 & - & - & - & 37.15 \\
\hline Sakleshpur-1 & - & - & - & 0 & Sakleshpur-3 & - & - & - & 0 \\
\hline Sakleshpur-2 & - & - & + & 33.54 & Siddapura-1 & - & - & - & 0 \\
\hline Sakleshpur-3 & + & + & + & 26.88 & Siddapura-2 & - & + & + & 30.73 \\
\hline Siddapura-1 & - & + & + & 28.54 & Siddapura-3 & - & - & - & 0 \\
\hline Siddapura-2 & - & - & - & 37.57 & Somavarapete-1 & - & - & - & 38.38 \\
\hline Siddapura-3 & + & + & + & 27.09 & Somavarapete-2 & - & - & - & 0 \\
\hline Somavarapete-1 & - & - & + & 34.67 & & & & & \\
\hline Somavarapete-2 & + & + & + & 25.32 & & & & & \\
\hline
\end{tabular}

